\title{
Focal Adhesion Kinase Promotes Integrin Adhesion Dynamics Necessary for Chemotropic Turning of Nerve Growth Cones
}

\author{
Jonathan P. Myers ${ }^{1,2,3}$ and Timothy M. Gomez ${ }^{1,2,3}$ \\ ${ }^{1}$ Department of Neuroscience, ${ }^{2}$ Medical Scientist Training Program, and ${ }^{3}$ Neuroscience Training Program, University of Wisconsin, Madison, Wisconsin \\ 53706
}

The ability of extending axons to navigate using combinations of extracellular cues is essential for proper neural network formation. One intracellular signaling molecule that integrates convergent signals from both extracellular matrix (ECM) proteins and growth factors is focal adhesion kinase (FAK). Analysis of FAK function shows that it influences a variety of cellular activities, including cell motility, proliferation, and differentiation. Recent work in developing neurons has shown that FAK and Src function downstream of both attractive and repulsive growth factors, but little is known about the effectors or cellular mechanisms that FAK controls in growth cones on ECM proteins. We report that FAK functions downstream of brain-derived neurotrophic factor (BDNF) and laminin in the modulation of point contact dynamics, phosphotyrosine signaling at filopodial tips, and lamellipodial protrusion. BDNF stimulation accelerates paxillincontaining point contact turnover and formation. Knockdown of FAK function either with a FAK antisense morpholino or by expression of FRNK, a dominant-negative FAK isoform, blocks all aspects of the response to BDNF, including the acceleration of point contact dynamics. On the other hand, expression of specific FAK point mutants can selectively disrupt distinct aspects of the response to BDNF. We also show that growth cone turning depends on both signaling cascades tested here. Finally, we provide the first evidence that growth cone point contacts are asymmetrically regulated during turning to an attractive guidance cue.

\section{Introduction}

The proper development of the nervous system is a complex process, requiring neuronal differentiation, migration, and guidance of extending axons. Imprecise neural network formation can lead to profound neurological disorders, such as mental retardation, epilepsy, autism, and schizophrenia (Yaron and Zheng, 2007). Axon guidance is mediated by growth cones, sensory-motile structures that integrate attractive and repulsive molecular cues to direct axons to their synaptic targets (TessierLavigne and Goodman, 1996; Yu and Bargmann, 2001). Guidance cues activate signals that regulate numerous cellular processes that growth cones have in common with motile cells, including protrusion of lamellipodia and filopodia (Huber et al., 2003; Lowery and Van Vactor, 2009).

A subsequent key step that controls cell motility is the stabilization of new protrusions through integrin receptor-mediated adhesion to the extracellular matrix (ECM). Growth cone adhesion to ECM proteins is a poorly understood process that occurs at specialized sites called point contacts, which are analogous to

Received May 12, 2011; revised June 30, 2011; accepted July 29, 2011.

Author contributions: J.P.M. and T.M.G. designed research; J.P.M. performed research; J.P.M. analyzed data; J.P.M. and T.M.G. wrote the paper.

This work was supported by NIH Grant NS41564 to T.M.G. We thank Kate Kalil and members of the Gomez laboratory for comments on the manuscript.

Correspondence should be addressed to Timothy M. Gomez, University of Wisconsin, 257 Bardeen Labs-SMI, 1300 University Avenue, Madison, WI 53706. E-mail: tmgomez@wisc.edu.

DOI:10.1523/JNEUROSCI.2381-11.2011

Copyright $\odot 2011$ the authors $\quad 0270-6474 / 11 / 3113585-11 \$ 15.00 / 0$ fibroblast focal adhesions (Gomez et al., 1996; Renaudin et al., 1999). While the regulation of cell motility by focal adhesions is well known (Mitra et al., 2005; Schaller, 2010), relatively few studies have examined the function of point contacts in growing axons. Growth cones form point contacts that contain a number of focal adhesion-related molecules, including focal adhesion kinase (FAK), paxillin, vinculin, and $\beta 1$-integrins (Gomez et al., 1996; Renaudin et al., 1999; Robles and Gomez, 2006). FAK is a particularly intriguing component of point contacts, as this signaling/scaffolding kinase regulates cell motility, together with its common signaling partner Src, by integrating signals from ECM proteins and growth factors (Mitra et al., 2005; Tomar and Schlaepfer, 2009). Interestingly, FAK and Src function downstream of both growth-promoting and -inhibiting axon guidance cues and regulate axon pathfinding in vivo. For example, FAK is essential for Netrin signaling (Li et al., 2004; Liu et al., 2004; Ren et al., 2004) and functions downstream of the inhibitory factors Sema3a (Bechara et al., 2008) and Ephrin-A1 (Woo et al., 2009). Previous work from our laboratory showed that FAK functions in normal pathfinding by peripheral Rohan-Beard sensory processes and midline crossing by spinal commissural axons in vivo (Robles and Gomez, 2006). Consistent with a bifunctional role in adhesion dynamics, FAK is involved in the disassembly of point contacts during Sema3a-induced growth cone collapse (Bechara et al., 2008) and the overstabilization of point contacts by retinal ganglion cells (RGCs) in response to Ephrin-A1 (Woo et al., 2009). The molecular basis for these diverse functions of FAK remain poorly understood in growth cones. 
We hypothesize that FAK regulates growth cone point contacts downstream of axon guidance cues and that proper axon pathfinding depends on coordination of protrusion and adhesion by FAK. We report that FAK is necessary for multiple aspects of the morphological effects of brain-derived neurotrophic factor (BDNF) on growth cones. We show that FAK controls protrusion and adhesion downstream of BDNF through distinct tyrosine residues and that both pathways are necessary for attractive turning. Additionally, we show that point contacts can be asymmetrically regulated across growth cones exposed to a BDNF gradient, key evidence that growth cone adhesion functions to direct axon guidance.

\section{Materials and Methods}

Expression constructs, embryo injection, and culturing. Expression constructs were subcloned into the Xenopus-preferred pCS2 vector for mRNA synthesis (Dave Turner, University of Michigan, Ann Arbor, MI). cDNA for chicken paxillin-GFP was provided by A. F. Horwitz (University of Virginia, Charlottesville, VA). GFP-FRNK was provided by P. Keely (University of Wisconsin, Madison, WI). YFP-dSH2 was provided by Benjamin Geiger (Weizmann Institute, Rehovot, Israel) and subcloned into CS2-GFP and CS2-mCherry ( $\mathrm{mCh}$ ) vectors. FAK Y397F, Y861F, and Y925F point mutants were provided by M. Schaller (West Virginia University, Morgantown, WV). A Xenopus FAK morpholino (FAK-MO) was designed and purchased from Gene Tools as previously described (Robles and Gomez, 2006). Xenopus laevis embryos were obtained as described previously (Gomez et al., 2003) and staged according to Nieuwkoop and Faber (1994). For direct expression experiments using GFP-FRNK, GFP-FAK, or FP-dSH2, two dorsal blastomeres of eight-cell-stage embryos were injected with $0.25-0.5 \mathrm{ng}$ of in vitro-transcribed, capped mRNA (mMessage Machine, Ambion) or 75100 pg of DNA for paxillin-GFP, mCh-FAK Y397F, Y861F, or Y925F. For FRNK point contact experiments, $0.3 \mathrm{ng}$ of myc-FRNK and $0.25 \mathrm{ng}$ of mCh-dSH2 RNA were injected with 75 ng of paxillin-GFP DNA. For morpholino experiments, we injected 1-2 nl of $1 \mathrm{~mm}$ FAK-MO combined with $\mathrm{mCh}$ dSH2 and/or paxillin-GFP DNA. Neural tube explant cultures containing a heterogeneous population of spinal neurons were prepared as previously described (Gomez et al., 2003). Explants were plated onto acid-washed coverslips coated with $25 \mathrm{mg} \cdot \mathrm{ml}^{-1}$ laminin (LN) (Sigma). Cultures were imaged or fixed 16-24 h after plating. All methods were approved by the University of Wisconsin School of Medicine Animal Care and Use Committee.

Image acquisition and analysis. For both live and fixed fluorescence microscopy, high-magnification images were acquired using either a $60 \times / 1.45$ NA objective lens on an Olympus Fluoview 500 laser-scanning confocal system mounted on an AX-70 upright microscope or a $100 \times 1$ 1.5 NA objective lens on a Nikon total internal reflection fluorescence (TIRF) microscope. On the confocal, samples were imaged at $2-2.5 \times$ zoom (pixel size $=165-200 \mathrm{~nm}$ ). Images were captured at $10-15 \mathrm{~s} \mathrm{in}$ tervals for $20 \mathrm{~min}$ before and $20 \mathrm{~min}$ after BDNF stimulation. For bright- field time-lapse microscopy, low-magnification phase-contrast images were acquired using a $20 \times$ objective on a Nikon microscope equipped with an $x-y$ motorized stage for multipositional imaging. Multipositional images were capture at $1 \mathrm{~min}$ intervals for $30 \mathrm{~min}$ before and 30 min after BDNF stimulation. Rate of outgrowth was calculated by drawing a single line from the growth cone leading edge at the beginning to the leading edge at the end of each period, divided by the time of each period. Live explant cultures were sealed within perfusion chambers as described previously (Gomez et al., 2003) to allow rapid exchange of solutions. Images were analyzed using ImageJ software (W. Rasband, National Institutes of Health, Bethesda, MD). Point contacts were identified as discrete areas containing paxillin-GFP that were at least two times brighter than the surrounding background and remained fixed in place for a minimum of $30 \mathrm{~s}$ (Woo and Gomez, 2006). The determination of proximal versus distal for Figure 8 was made using a line extending from the center of the axon $5 \mu \mathrm{m}$ distal to the growth cone through the center of 

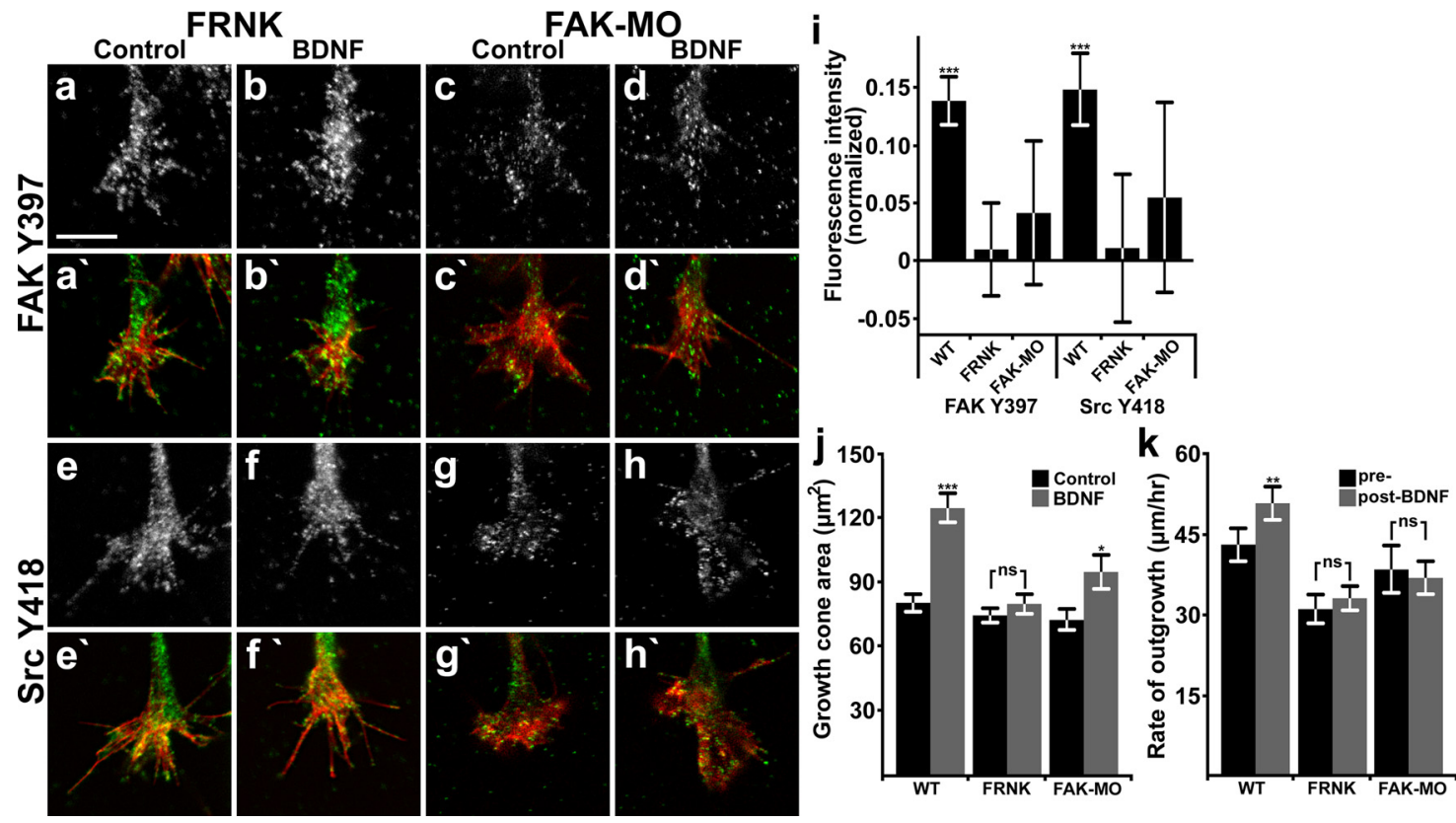

Figure 2. FAK is necessary for Src activation and the functional effects of BDNF on growth cone motility. $\boldsymbol{a}-\boldsymbol{h}$, Representative images of neurons expressing dominant-negative FAK (FRNK; $\boldsymbol{a}, \boldsymbol{b}$, $\boldsymbol{e}, \boldsymbol{f})$ or loaded with FAK-MO $(\boldsymbol{c}, \boldsymbol{d}, \boldsymbol{g}, \boldsymbol{h})$, that were treated with control media or BDNF and immunostained with phospho-specific antibodies to the sites indicated. $\boldsymbol{a}^{\prime}-\boldsymbol{h}^{\prime}$, Immunolabeled images $(\boldsymbol{a}-\boldsymbol{h}$; green) are merged with fluorescent phalloidin labeling of F-actin (red). $\boldsymbol{i}$, Quantification of fluorescence intensity in growth cones stimulated for 5 min with $100 \mathrm{ng} / \mathrm{ml}$ BDNF, normalized to unstimulated growth cones. $n \geq 40$ for each antibody and condition. Data from Figure $1 i$ are shown for comparison. $\boldsymbol{j}$, Growth cone area measured from thresholded phalloidin labeling. $\boldsymbol{k}$, Rate of neurite outgrowth measured by time-lapse phase contrast imaging. Note that data duplicated from Figure $1 k$ are included for comparison. Significance determined by Student's $t$ test to unstimulated neurons, paired analysis in $\boldsymbol{k}^{* * *} p<0.001,{ }^{* *} p<0.01,{ }^{*} p<0.05 . n \geq 24$ for each condition. Scale bar, $10 \mu \mathrm{m}$.

mass of the fluorescent signal. Kymographs were created using Image from 1-pixel-wide lines. Measurements of pY-FAK and pY-Src intensity were made by first selecting the perimeter of growth cones from thresholded F-actin-labeled images based on intensity to exclude background using ImageJ. These user-defined regions were then used to measure the average pixel intensity of pY labeling within nonthresholded growth cones. For display purposes, some images were pseudocolored using ImageJ lookup tables. For all figures, images were processed in Adobe Photoshop (Adobe Systems) as follows: brightness levels were adjusted, an unsharp mask routine was applied to improve edge detection, and the images were converted to 8 bit depth and cropped. Statistical significance for point contact lifetime and adhesion frequency in Figures 4-6 was determined using two-way repeated-measure ANOVA on all genetic conditions. Significance for adhesion density was determined by Student's $t$ test, since no comparisons were made across genetic conditions. Variance reported as \pm SEM from at least two independent experiments using Prism software (GraphPad Software).

Dynamic adhesion maps. Dynamic adhesion map images were prepared from image stacks as detailed previously (Santiago-Medina et al., 2011). Briefly, an image stabilization algorithm was applied, and an unsharp mask routine was applied, to improve edge detection, followed by thresholding to highlight the puncta of interest. An 8 bit binary filter was applied to equalize point contact intensities. Image stacks were then converted to 16 bit and user-defined subsets were summed so that intensity encodes pixel lifetime. Final images were contrast enhanced and pseudocolorized.

Growth cone turning assays. Most turning assays were performed essentially as described previously (Ming et al., 1997b) using a 40× air objective on a Zeiss Axiovert microscope equipped with a Coolsnap HQ CCD camera (Roper Scientific). Briefly, micropipettes with a tip diameter of $1 \mu \mathrm{m}$ were filled with either $1 \times$ modified Ringer's culture medium or medium containing $10 \mu \mathrm{g} \cdot \mathrm{ml}^{-1}$ BDNF. Micropipettes were positioned at a distance of $100 \mu \mathrm{m}$ from the growth cone leading edge at $45^{\circ}$ with respect to the neurite axis. An electronically gated pressure application system (World Precision Instruments) controlled by a programmable pulse generator (Grass 48 Stimulator, Grass Technologies) to generate 50 ms pulses with a positive pressure of $8 \mathrm{psi}$ at a frequency of $1 \mathrm{~Hz}$. Acute turning while performing point contact imaging was performed with a $100 \times$ oil objective on a Nikon TIRF microscope as described above. Micropipettes with a tip diameter of $1 \mu \mathrm{m}$ were filled with $10 \mu \mathrm{g} \cdot \mathrm{ml}^{-1}$ BDNF and positioned $100-130 \mu \mathrm{m}$ from the growth cone leading edge at $60-90^{\circ}$ with respect to the neurite axis. A digital pressure application system (Femtojet, Eppendorf) applying continuous pressure was set to 1-2 psi and lowered at the beginning of each assay. During each assay, images were collected using MetaMorph acquisition software (Universal Imaging). Only growth cones that extended at least $10 \mu \mathrm{m}$ during imaging were included in our analysis. Turning angles were determined using ImageJ software (W. Rasband, National Institutes of Health, Bethesda, $\mathrm{MD}$ ) by measuring the angle of the $5 \mu \mathrm{m}$ distal segment of the neurite at the beginning and end of each trial.

Immunocytochemistry. For immunocytochemistry (ICC), spinal neuron cultures were fixed in $4 \%$ paraformaldehyde in Krebs + sucrose fixative (4\% PKS) (Dent and Meiri, 1992), permeabilized with $0.1 \%$ Triton X-100, and blocked in 1.0\% fish gelatin in CMF-PBS for $1 \mathrm{~h}$ at room temperature. Primary antibodies were used at the following dilutions in blocking solution: 1:500 all pY-FAK antibodies (Biosource) and 1:500 all pY-Src antibodies (Biosource). Alexa-Fluor-conjugated secondary antibodies were purchased from Invitrogen and used at 1:250 in blocking solution. Included with secondary antibodies was Alexa-647 phalloidin (1:50-1:100; Invitrogen) to label filamentous actin (F-actin).

\section{Results}

BDNF signals through FAK and Src to induce growth cone morphological changes and accelerate neurite outgrowth

To begin to test how FAK and Src may integrate growth factor and integrin signaling, we cultured Xenopus spinal neurons on $\mathrm{LN}$, an integrin receptor ligand known to activate intracellular signals and promote axon outgrowth (Letourneau et al., 1994; Hynes, 2002). Neurons on LN were stimulated with $100 \mathrm{ng} / \mathrm{ml}$ BDNF for $5 \mathrm{~min}$, fixed, and labeled by ICC with phosphotyrosine-specific antibodies to FAK and Src. Growth cones were imaged by confocal microscopy to assess both morphological changes and quantify the phosphorylation of several key tyrosine 


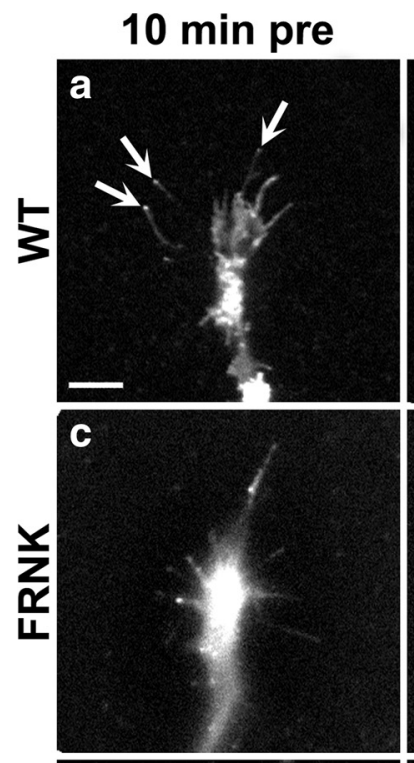

10 min BDNF
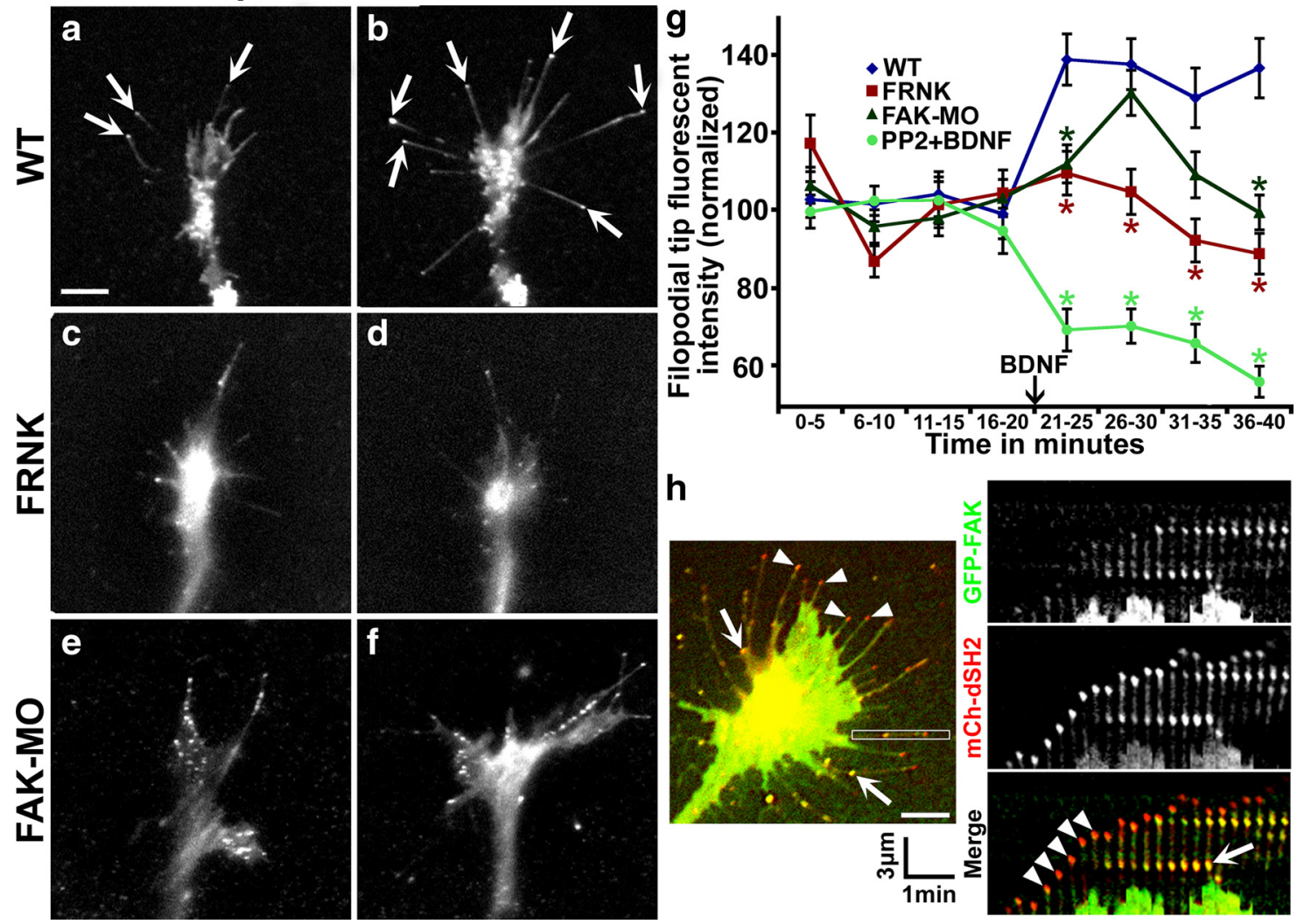

Figure 3. FAK is necessary for Src-mediated tyrosine phosphorylation at filopodial tips. $\boldsymbol{a}$ - $\boldsymbol{f}$, Time-lapse TIRF microscopy images of mCh-dSH2-expressing growth cones on LN 10 min before ( $\boldsymbol{a}$, $\boldsymbol{c}, \boldsymbol{e})$ and 10 min during $(\boldsymbol{b}, \boldsymbol{d}, \boldsymbol{f})$ BDNF stimulation. Note a robust increase in fluorescence at filopodial tips (arrows) in wild-type neurons $(\boldsymbol{a}, \boldsymbol{b})$, but not neurons coexpressing GFP-FRNK (c, $\boldsymbol{d}$ ) or loaded with FAK-MO $(\boldsymbol{e}, \boldsymbol{f}) . \boldsymbol{g}$, Average fluorescent intensity at filopodial tips in wild-type, FRNK, and FAK-MO neurons binned over 5 min intervals during stimulation with BDNF. Note a sustained increase in fluorescent PY signals at filopodial tips of wild-type growth cones after BDNF, but a failure to increase or sustain filopodial tip intensity in FAK LOF neurons. $n \geq 20 \mathrm{growth}$ cones and 750 filopodial tips per condition. $\boldsymbol{h}$, A single time point of a live growth cone expressing GFP-FAK and $\mathrm{mCh}$-dSH2 (left panel). Right panels show a time series of single channel and merged images generated from the boxed region indicated on the left panel at $15 \mathrm{~s}$ intervals. Point contacts (arrows) show colocalization of FAK and dSH2 and appear yellow in merge, while FAK does not localize to the growing PY-positive tips (arrowheads). Scale bars, $5 \mu \mathrm{m}$.

residues on FAK and Src. Activation of FAK and Src is associated with autophosphorylation at Y397 and Y418, respectively (Schaller et al., 1994; Thomas and Brugge, 1997). BDNF increased the levels of FAK pY-397 and Src pY-418 relative to unstimulated control neurons (Fig. $1 a, b, e, f, i$ ), suggesting that BDNF activates these kinases in growth cones on LN. Subsequently, phosphorylation of the Y861 site on FAK by Src creates a binding site for several downstream targets, including p130Cas, which can activate Rac1 via DOCK180 (Bouton et al., 2001). Stimulation by BDNF also led to an increase in pY-861 FAK (Fig. $1 c-d, i)$, indicating that this pathway is downstream of TrkB signaling. As a negative regulator of Src, Csk phosphorylates Y529 of Src, which promotes an autoinhibited confirmation of Src (Okada and Nakagawa, 1989; Thomas and Brugge, 1997). Consistent with Src activation by BDNF, we find that pY-529 Src is reduced in growth cones (Fig. $1 g-i$ ). Opposite changes in the phosphorylation of specific tyrosine residues on Src detected by ICC also serves as a useful control for any potential artifact due to morphological changes in growth cones. Growth cone area was measured from thresholded F-actin labeling with fluorescent phalloidin, which showed that BDNF treatment increased the average growth cone area by $>50 \%$ (Fig. $1 j$ ).

To assess the acute effects of BDNF on growth cone behavior, we treated neurons on $\mathrm{LN}$ with BDNF during time-lapse phase contrast imaging. We found that BDNF induces a sustained in- crease in the rate of neurite outgrowth concomitant with veil expansion. The average rate of outgrowth by all neurons imaged increased by $18.6 \pm 6.0 \%$ (Fig. $1 k$ ). This relatively modest acceleration is likely due to the high basal rate of outgrowth of neurons on LN, which is probably due to high FAK and Src activities (Robles and Gomez, 2006). In addition, based on the morphological response, it appears that not all the neurons present in Xenopus spinal cultures respond to BDNF. Approximately $60 \%$ of the neurons observed in our outgrowth assay showed the characteristic lamellipodial expansion associated with BDNF stimulation (Ming et al., 1997a). If only neurons that exhibit clear morphological changes in response to BDNF are included, neurite outgrowth accelerates by $48.6 \pm 10.0 \%$ (from $47.4 \mu \mathrm{m} / \mathrm{h}$ to $70.4 \mu \mathrm{m} / \mathrm{h}$ ). A population of spinal neurons unresponsive to BDNF is consistent with previous studies (Robles and Gomez, 2006) and indicates that the phosphorylation changes we measured (Fig. 1) are likely dampened by this BDNF-insensitive population of neurons.

To test whether FAK function is necessary for the effects of BDNF on growth cone behavior, we inhibited FAK function by two means. First, we used a translation blocking antisense morpholino oligomer that reduces FAK expression as previously described (xFAKmo; Robles et al., 2006). Second, we expressed a dominant-negative isoform of FAK termed FAK-related nonkinase [FRNK (Schaller et al., 1993)]. Measurement of pY-397 FAK 

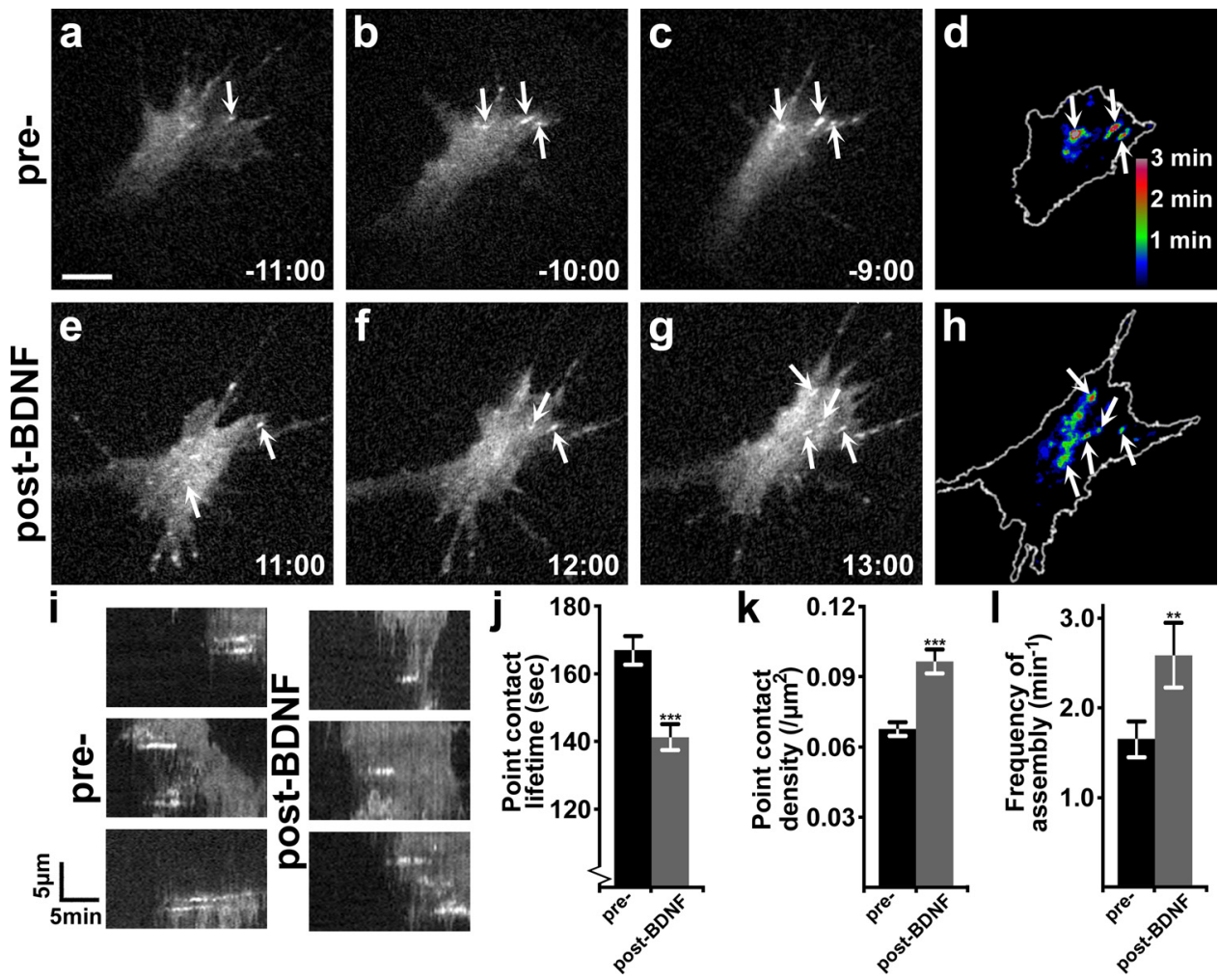

Figure 4. BDNF stimulates growth cone point contact assembly and turnover. $\boldsymbol{a}-\boldsymbol{c}$, Time-lapse TIRF microscopy images at 1 min intervals of paxillin-GFP-expressing spinal neuron growth cone on LN. Note several point contacts at the leading edge containing paxillin-GFP that appear in consecutive frames (arrows). $\boldsymbol{d}$, Dynamic adhesion map image illustrating point contact lifetimes constructed by summation of 5 min (20 images total) spanning time points in $\boldsymbol{a}-\boldsymbol{c}$. Note several long lifetime point contacts (arrows). $\boldsymbol{e}-\boldsymbol{g}$, Still images from the same neuron as in $\boldsymbol{a}-\boldsymbol{d}$, stimulated with $100 \mathrm{ng} / \mathrm{ml}$ BDNF. Note formation of many new point contacts (arrows), and that many disappear. $\boldsymbol{h}$, Dynamic adhesion map of point contact lifetime summed over 5 min during BDNF stimulation (e- $\boldsymbol{g}$ ). Note cooler-colored point contacts indicating shorter lifetimes, as well as an increased number of point contacts. $\boldsymbol{i}$, Kymographs generated from the time series used in $\boldsymbol{a}-\boldsymbol{h}$ before and after BDNF addition. Note the shorter lifetime adhesions after BDNF addition. $j-I$, Quantification of point contact dynamics shows a decreased lifetime $(\boldsymbol{l})$ and increased density $(\boldsymbol{k})$ and frequency of assembly $(\boldsymbol{I})$ with BDNF. See Materials and Methods for determination of significance. $n \geq 99$ point contacts for $\boldsymbol{j}$, and $n=10$ growth cones for $\boldsymbol{k}$ and $\boldsymbol{I} .{ }^{* * *} p<0.001,{ }^{* *} p<0.01$. Scale bar, $5 \boldsymbol{\mu m}$ in $\boldsymbol{a}-\boldsymbol{h}$ or as indicated in kymograph.

and pY-418 Src by ICC showed that FAK loss of function (LOF) decreased the basal activity of both kinases (data not shown), and blocked their activation by BDNF (Fig. $2 a-i$ ). These results indicate that Src activity is dependent on FAK function in growth cones. Moreover, the Src-specific inhibitor PP2 also blocked FAK Y397 and Src Y418 phosphorylation in response to BDNF (data not shown), suggesting that these kinases activate cooperatively. Consistent with the loss of FAK and Src activities, we find that BDNF stimulation of membrane protrusion, as assessed by changes in growth cone area, as well as neurite acceleration, are both inhibited when FAK signaling was disrupted (Fig. 2j,k). These results indicate that FAK and Src are both necessary for basal axon outgrowth on LN, as well as stimulated outgrowth by BDNF.

FAK is necessary for Src-mediated tyrosine phosphorylation at filopodial tips

Previously we showed by imaging phosphotyrosine (PY) signals of live neurons on poly-D-lysine (PDL) that BDNF activates Src, a kinase commonly coactivated with FAK, which increases PY at the tips of growth cone filopodia (Robles et al., 2005). To image PY signals in living cells, we express a biosensor consisting of mCherry $(\mathrm{mCh})$ fused to a tandem dimer of the $\mathrm{SH} 2$ domain (dSH2) from c-Src (Kirchner et al., 2003). As described previously, this reporter detects Src-dependent tyrosine phosphorylation (Robles et al., 2005). To determine the effects of integrin engagement on PY signaling downstream of BDNF, we cultured mCh-dSH2-expressing neurons on LN and performed time- lapse TIRF microscopy. BDNF induced a strong and sustained increase in PY at filopodial tips (Fig. $3 a, b, g$ ), indicating that this aspect of the BDNF response is maintained with integrin engagement. Moreover, the effects of BDNF on filopodial tip PY was blocked by PP2, indicating that Src is still necessary for this aspect of the response to BDNF on LN. To test whether FAK function is also necessary for PY signals by Src phosphorylation at filopodia tips, we imaged mCh-dSH2 in FAK LOF neurons. Surprisingly, reducing FAK function with FRNK prevented the increased PY to filopodial tips in response to BDNF, while a partial, but unsustained response was seen in neurons loaded with FAK-MO (Fig. $3 c-g)$. This result indicates that FAK is necessary for Srcdependent PY signaling downstream of BDNF, which is unexpected, since active Src, but not FAK, localizes with PY at filopodia tips by ICC (Robles et al., 2005; Robles and Gomez, 2006). To test whether FAK and PY colocalize to growing filopodial tips, we imaged live neurons coexpressing GFP-FAK and mCh-dSH2. While FAK puncta localize to point contacts along filopodia, FAK was never detected at growing PY-positive filopodial tips (Fig. $3 h$ ). Together, these results suggest that Src is activated by FAK downstream of BDNF proximal to the tips of filopodia and active Src is subsequently recruited to filopodial tips.

BDNF promotes new point contact assembly and adhesion turnover

We previously found that inhibitory axon guidance cues can reduce axon outgrowth by either disrupting or overstabilizing growth cone point contacts. To determine whether BDNF may 

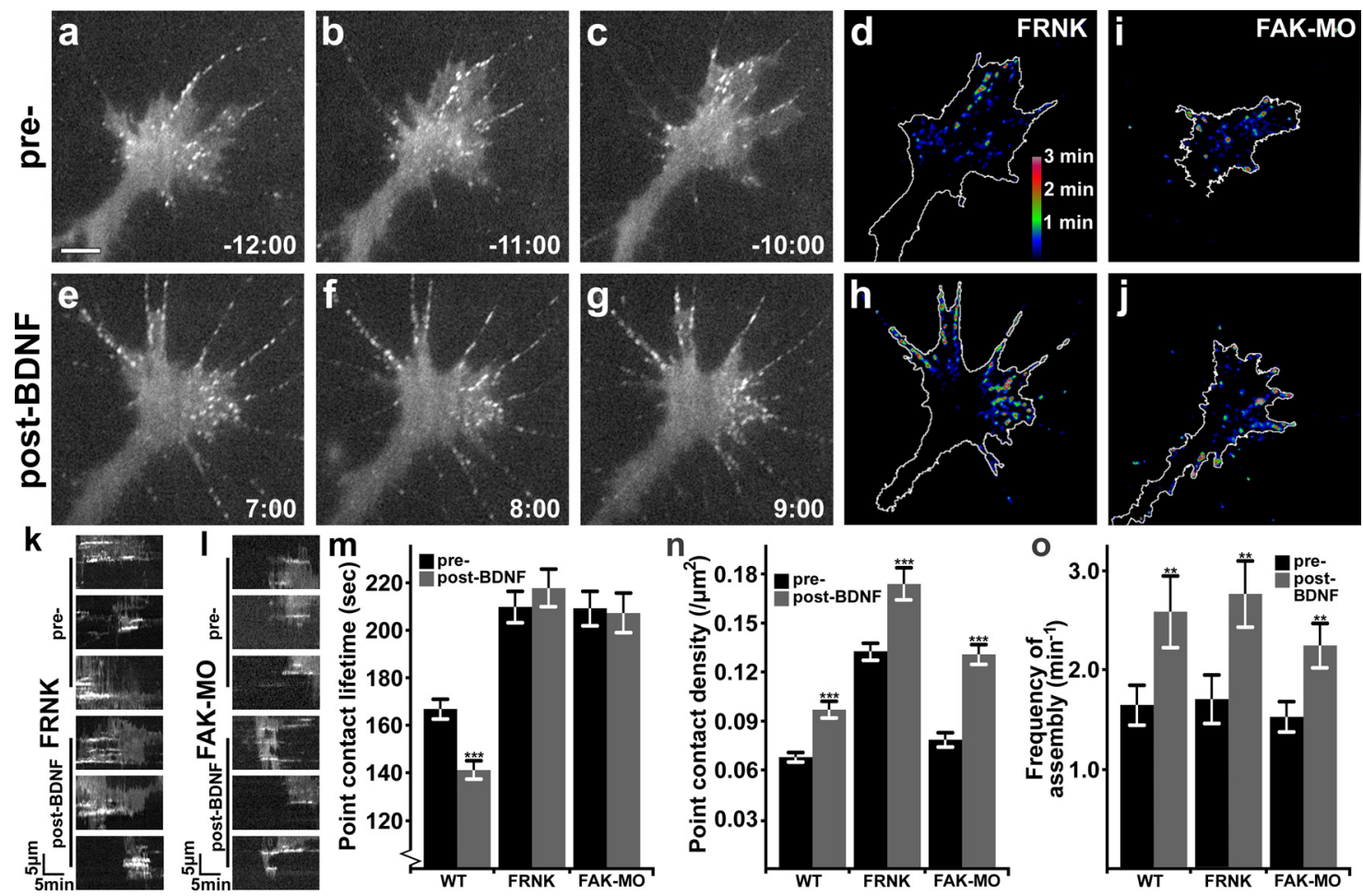

Figure 5. FAK knockdown disrupts turnover but not formation of point contacts. $\boldsymbol{a}-\boldsymbol{c}$, Time-lapse TIRF microscopy images at 1 min intervals of a FRNK and paxillin-GFP coexpressing spinal neuron growth cone on LN. Note the decreased protrusive activity and increased number of point contacts, as well as their stability from frame to frame. $d$, Dynamic adhesion map of 5 min summed frames (including $\boldsymbol{a}-\boldsymbol{c}$ ) before BDNF stimulation. $\boldsymbol{e}-\boldsymbol{g}$, Growth cone from $\boldsymbol{a}-\boldsymbol{d}$, stimulated with $100 \mathrm{ng} / \mathrm{ml}$ BDNF. Note the absence of increased protrusion, the increase in paxillin-GFP puncta, and the maintained persistence across frames. $\boldsymbol{h}$, Dynamic adhesion map during BDNF stimulation. $\boldsymbol{i}, \boldsymbol{j}$, Dynamic adhesion maps from a FAK-MO loaded growth cone, before $(\boldsymbol{i})$ and during $(\boldsymbol{j})$ BDNF stimulation generated as in $\boldsymbol{d}$ and $\boldsymbol{h} . \boldsymbol{k}, \boldsymbol{I}$, Kymographs generated from paxillin-GFP-labeled growth cones coexpressing FRNK (k) or loaded with FAK-MO (I) before and after BDNF. Note there is little change in lifetime of adhesions after BDNF addition. $\boldsymbol{m}-\boldsymbol{0}$, Quantification of point contact dynamics shows longer point contact lifetimes in FAK LOF neurons, but no change with BDNF ( $\boldsymbol{m}$ ). On the other hand, point contact density $(\boldsymbol{n})$ and frequency of formation (o) increase significantly with BDNF. Note that data duplicated from Figure $4 j-I$ are included for comparison. See Materials and Methods for determination of significance. $n \geq 94$ point contacts for $\boldsymbol{m}$, and $n \geq 10$ neurons for each condition in $(\boldsymbol{n}, \boldsymbol{0}) .{ }^{* * *} p<0.001,{ }^{* *} p<0.01$ compared to unstimulated condition. Scale bar, $5 \mu \mathrm{m}$ in $\boldsymbol{a}-\boldsymbol{j}$ or as indicated in kymographs.

enhance neurite outgrowth through modulation of point contacts, we imaged paxillin-GFP in live growth cones during stimulation with BDNF (Fig. 4). Paxillin is an adaptor protein that has important regulatory control over integrin-mediated adhesion (Turner, 2000), and paxillin-GFP has previously been shown to be a reliable marker for growth cone point contacts (Robles and Gomez, 2006; Woo and Gomez, 2006; Woo et al., 2009). We imaged live paxillin-GFP-expressing growth cones on LN by time-lapse TIRF microscopy before and during stimulation with $100 \mathrm{ng} / \mathrm{ml}$ bath application of BDNF. Before BDNF simulation, point contacts appear as relatively stable puncta that assemble and disassemble near the leading edge with an average lifetime of $\sim 3$ min (Fig. 4i). Acute stimulation with BDNF has two important effects on growth cones as they expand due to increased protrusive activity (Fig. 2). First, point contact lifetime decreases with BDNF stimulation (Fig. $4 i, j$ ), indicating that BDNF promotes point contact turnover, a regulatory event that may be necessary for the increase in rate of outgrowth. Although point contacts turn over more rapidly, their density increases (Fig. $4 k$ ) suggesting that BDNF also promotes point contact formation. The frequency of new point contact assembly increases by $57.3 \pm$ $22.0 \%$ (Fig. $4 l$ ). To visualize changes in the number and lifetime of point contacts in a single image before and after BDNF treatment, we developed a method to display adhesion lifetime according to fluorescent intensity (see Materials and Methods). Briefly, individual fluorescent images are thresholded to reveal adhesions and made 8 bit binary. Binary images are then summed for equivalent periods before and after treatment and a color lookup table is applied so intensity encodes point contact lifetime (Fig. $4 d, h$ ). Comparing these dynamic adhesion map images collected before and after stimulation with BDNF shows a few warm-colored point contacts before treatment and more coolercolored point contacts after BDNF treatment, indicating accelerated point contact turnover.

\section{FAK knockdown disrupts turnover but not formation of point contacts}

To assess the role of FAK in the control of point contact dynamics, we imaged paxillin-GFP in FAK LOF neurons by time-lapse TIRF microscopy (Fig. 5). In both FRNK-expressing and FAK-MO growth cones, we found that the density and persistence of point contacts during basal outgrowth on LN was elevated (Fig. 5). Slow adhesion turnover in FAK LOF neurons is consistent with previous findings in retinal ganglion neurons (Woo et al., 2009) and may be responsible for the reduced basal rate of outgrowth (Fig. $2 k$ ). Interestingly, BDNF stimulation of FAK LOF neurons led to an increase in the density of point contacts similar to wild-type neurons, but no change in adhesion lifetime. Increased point contact density with no apparent effect on lifetime is evident in dynamic adhesion map images of both FRNK and FAK MO neurons (Fig. $5 d, h, i, j)$. Measurement of point contact dynamics shows a significantly longer point contact lifetime in FAK LOF neurons, but no significant change in turnover after BDNF treatment (Fig. $5 k-m$ ). The increase in lifetime 

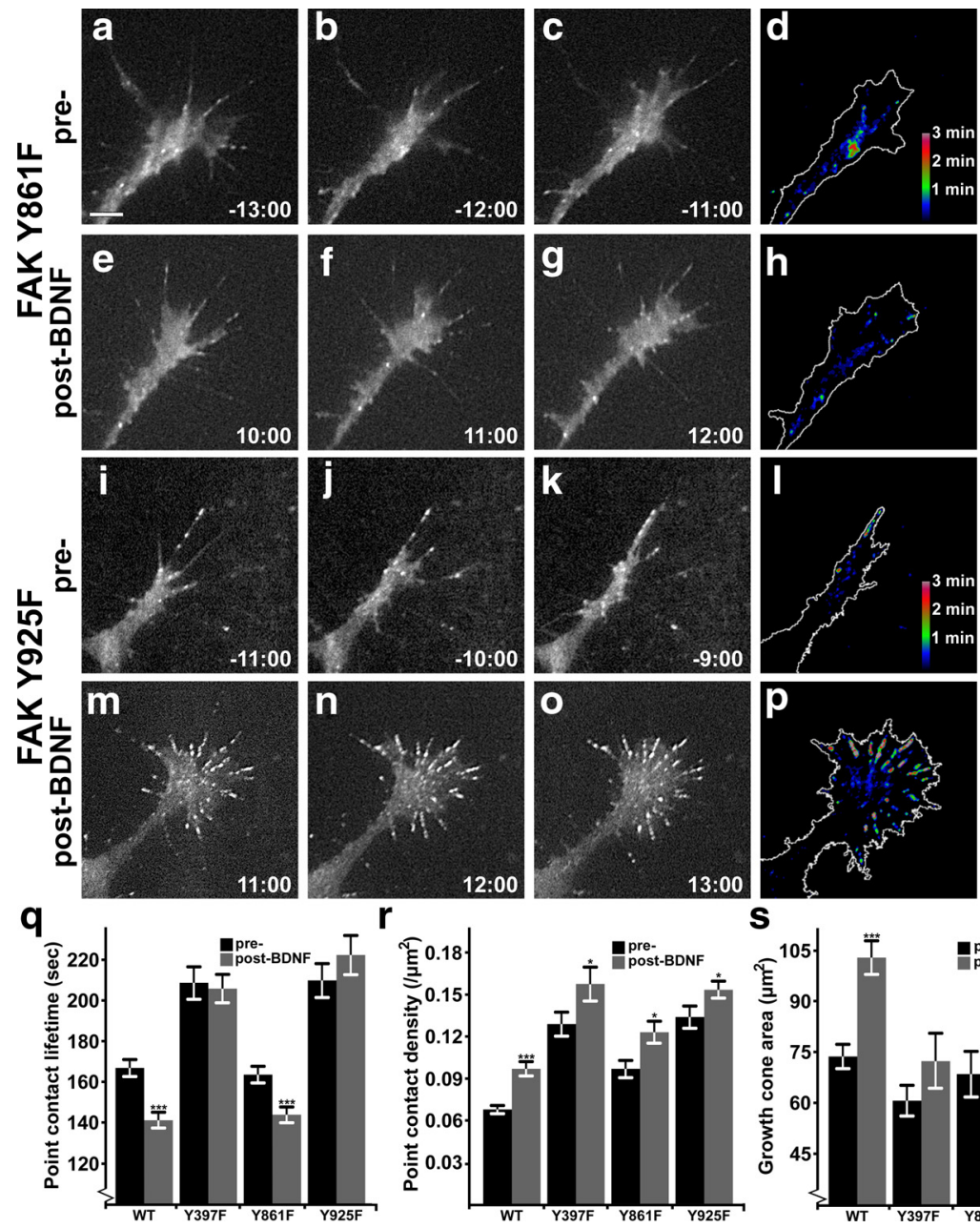

$\mathbf{S}$

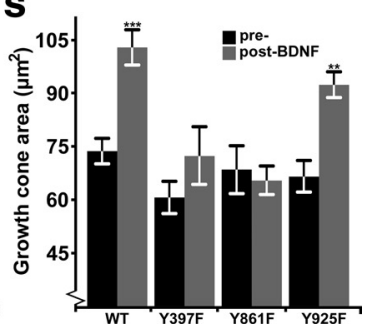

Figure 6. FAK point mutants selectively disrupt distinct aspects of BDNF signaling. $\boldsymbol{a}-\boldsymbol{h}$, Time-lapse TIRF microscopy images at 1 min intervals of a FAK Y861F and paxillin-GFP coexpressing spinal neuron growth cone on LN before and during BDNF stimulation. In the still frames, note the lack of protrusive activity with BDNF stimulation, with maintained point contact dynamics relative to wild type. Note in the dynamic adhesion map images $(\boldsymbol{d}, \boldsymbol{h})$, a decrease in point contact lifetime is apparent with little change of growth cone area. $\boldsymbol{i}-\boldsymbol{p}$, Still images and dynamic adhesion maps of a FAK Y925F and paxillin-GFP-coexpressing spinal neuron growth cone on LN before and during BDNF stimulation. Note a robust change in growth cone area with BDNF in both the still frames and in the dynamic adhesion map, together with the assembly of a number of highly stable point contacts $(\boldsymbol{p}) . \boldsymbol{q}, \boldsymbol{r}$, Quantification of point contact dynamics shows site-specific effects on adhesion lifetime $(\boldsymbol{q})$, but increased adhesion density $(\boldsymbol{r})$ in all mutants with BDNF. Note that data duplicated from Figure 4,j and $k$, are included for comparison. s, Quantification of growth cone area by thresholded fluorescent signal shows that the Y925F mutant does not block the effects of BDNF. See Materials and Methods for determination of significance. $n \geq 99$ point contacts for $\boldsymbol{q}$, and $n \geq 10$ neurons for each condition in $\boldsymbol{r}$ and $\boldsymbol{s}$. ${ }^{* * *} p<$ $0.001,{ }^{* *} p<0.01,{ }^{*} p<0.05$ compared to unstimulated condition. Scale bar, $5 \mu \mathrm{m}$.

was significant ( $p<0.001$, see Materials and Methods) compared to wild-type for each LOF condition in the basal and BDNFstimulated conditions. However, BDNF still promotes the assembly of new adhesions in FAK LOF neurons, resulting in a significant increase in point contact density (Fig. 5n). The frequencies of assembly were comparable with wild type for both FAK LOF conditions before and after BDNF treatment (Fig. $5 o$; $p>0.05$, see Materials and Methods). Inhibition of point contact turnover, together with a normal assembly rate, led to a dramatic increase in adhesion density with BDNF application. Note that adhesion density is higher in FRNK neurons compared to wildtype and FAK-MO. The consistently stronger phenotype observed in FRNK-expressing neurons is likely due to a more complete inhibition of FAK function. Together, these data suggest that BDNF stimulates neurite outgrowth on LN by enhancing the rate of FAK-independent point contact assembly, in coordination with FAK-mediated increased point contact turnover.

FAK tyrosine mutants disrupt specific aspects of BDNF signaling

Active FAK links to distinct downstream effectors through several key phosphorylated tyrosine residues. We tested tyrosine mutants of FAK to determine their roles in the control of growth cone motility in response to BDNF. Expressing tyrosine to phenylalanine mutants of FAK has previously been shown to interfere with binding of endogenous target proteins (Lim et al., 2004; Hamadi et al., 2005). We examined the effects of three key FAK tyrosine mutants, Y397F, Y861F, and Y925F, on growth cone behavioral outputs activated by FAK. The Y397F mutant blocks autoactivation of FAK, which prevents binding and activation of Src (Cary et al., 1996; Mitra et al., 2005). Src is responsible for phosphorylating additional tyrosine residues on FAK, including Y861 and Y925. According to published reports, we expected that the Y397F mutant would function as a dominant-negative protein similar to FRNK, preventing activation of downstream effectors due to a lack of FAK/Src cosignaling. Y861 of FAK associates with several signaling partners, particularly p130Cas, which can activate Rac1 and Cdc42. Importantly, the Y861 site has been shown to be essential for Netrin-dependent axon guidance (Liu et al., 2007). Last, phosphorylation at Y925 has been shown to be a key step for focal adhesion turnover in migrating fibroblasts (Brunton et al., 2005). Y925 FAK binds Grb2, which is believed to dissociate FAK from paxillin and disassemble adhesion complexes (Mitra et al., 2005; Scheswohl et al., 2008).

To test the roles of FAK in growth cones, we coexpressed mCherry-tagged FAK tyrosine mutants with paxillin-GFP and performed time-lapse TIRF microscopy during BDNF stimulation as described. As expected, the Y397F FAK mutant affected growth cones similarly to FRNK, with an increase in point contact lifetime and density at baseline. In addition, Y397F FAK prevented point contact changes in response to BDNF (Fig. 6q,r, $p<0.001$ for lifetime compared to wild-type, see Materials and Methods), as well as BDNF-driven membrane protrusion, as indicated by growth cone area (Fig. $6 s$ ). Interestingly, FAK Y861F did not significantly effect basal point contact lifetimes, nor prevent accelerated adhesion turnover in response to BDNF (Fig. $6 a-h, q$ ). However, Y861F FAK did block increased growth cone area after BDNF (Fig. 6s). With a slightly decreased area, this mutant also displayed a modest increase in point contact density (Fig. $6 r$ ). This finding suggests that BDNF stimulates multiple independent signaling pathways through FAK. The third tyrosine mutant we tested, FAK Y925F, also showed a distinct phenotype (Fig. 6i-p). Similar to Y397F, 
growth cones expressing Y925F FAK exhibited a slow basal rate of point contact turnover (Fig. 6q, $p<0.001$ compared to wild-type, see Materials and Methods), which did not change with BDNF stimulation. However, Y925F FAK did not prevent growth cone expansion by BDNF (Fig. 6s). Therefore, FAK Y925F appears to selectively prevent accelerated point contact turnover induced by BDNF, but can still transmit the signals necessary for lamellipodial expansion. All three point mutants tested had equivalent frequencies of formation of point contacts compared to wild type ( $p>0.05$, see Materials and Methods), confirming that FAK is not necessary for this aspect of BDNF signaling. Analysis of the rate of neurite outgrowth by neurons expressing these FAK mutants revealed that they exhibited comparable rates of outgrowth to FRNK-expressing neurites, but did not accelerate with BDNF application (data not shown). These results suggest that specific FAK tyrosine residues activate distinct signaling pathways downstream of BDNF.

\section{Chemoattraction to BDNF depends on} at least two distinct signaling pathways To determine how FAK Y861 and Y925 phosphorylation and subsequent signaling contribute to the guidance of growing neurons on LN, we performed turning assays using BDNF gradients. Neurons from wild-type embryos showed an attractive response to BDNF, turning an average of $17.8 \pm 5.0^{\circ}$ toward the pipette (Fig. $7 a, e$ ), but no significant response to a media control pipette $\left(0.3 \pm 3.8^{\circ}\right.$; Fig. $\left.7 b\right)$. Expression of either FAK Y861F or Y925F blocked attraction toward BDNF (turning angles of $1.3 \pm 2.4^{\circ}$ and $4.7 \pm 3.2^{\circ}$, respectively), but did not significantly slow axon outgrowth (Fig. $7 c-f$ ). This result shows that BDNF not only generates distinct signals and outputs mediated by FAK, but that both of the signals tested here are necessary for attractive turning. Note that as expected, not all neurons are attracted toward BDNF. From the scatter plot of turning angles (Fig. 7e), it is possible to distinguish the BDNF responsive and nonresponsive neurons tested. One population of neurons is clustered around $30^{\circ}$, while the others cluster around $0^{\circ}$, suggesting that nonresponsive neurons dampen the average response to BDNF.

\section{Point contacts are locally regulated during growth} cone turning

Our previous results showed that altering point contact dynamics with the Y925F FAK mutant inhibited growth cone turning to BDNF. However, this does not prove that differences in adhesion dynamics across growth cones promote attractive turning. To determine whether point contact number or lifetime are regulated asymmetrically across growth cones during turning, we performed time-lapse TIRF microscopy on paxillin-GFP and $\mathrm{mCh}-\mathrm{dSH} 2$ dual-expressing neurons exposed to a gradient of BDNF. The BDNF-loaded pipette was placed at a $90^{\circ}$ angle to the axon trajectory to maximize the signal differential observed across growth cones. Additionally, we quantified only those growth cones that exhibited an attractive response ( 9 of 16 neurons tested), to provide a more accurate measure of point contact changes in the BDNF-sensitive population. Importantly, we used the PY signals of the mCh-dSH2 channel to identify the responsive neurons. These neurons reoriented positively toward the pipette, or in some cases exhibited a sideways translocation toward the pipette. This side-stepping behavior may be due to the orientation of the gradient perpendicular to the direction of outgrowth.

Analysis of point contacts and PY signals show that both filopodia tips and adhesions are differentially promoted on the proximal side of growth cones toward the source of BDNF. Differential adhesions form rapidly, as seen by comparison with the first frame of this sequence (Fig. $8 a$ ) and as illustrated with dynamic adhesion map images generated from consecutive $3 \mathrm{~min}$ time periods during turning toward BDNF (Fig. $8 a-d$ ). The adhesion maps show both an increase in the number of point contacts (highlighted by white arrows) and a decrease in point contact lifetime on the side of the growth cone facing the BDNF gradient. Moreover, quantification of point contact lifetimes indicate a significantly faster turnover rate nearest the BDNF gradient (Fig. 8h), with values of $118.1 \pm 4.2 \mathrm{~s}$ proximal versus $149.5 \pm 5.9 \mathrm{~s}$ distal. The frequency of assembly on the proximal 

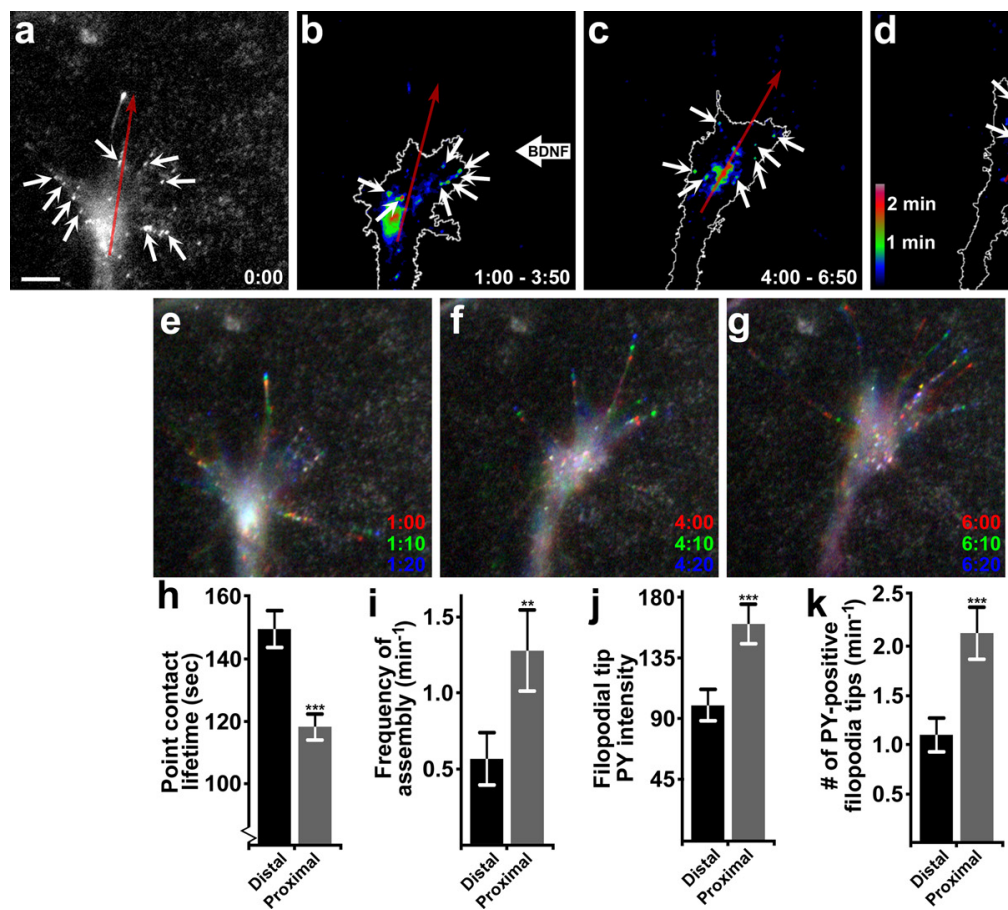

Figure 8. Point contacts are asymmetrically regulated during growth cone turning. $\boldsymbol{a}$, The first time point of a GFP-dSH2expressing neuron visualized by TIRF microscopy immediately before the BDNF gradient was applied. Point contacts are labeled with arrows, which appear symmetrically distributed across this growth cone. Red arrows indicate the divide between proximal and distal as well as the direction of growth. $\boldsymbol{b}$ - $\boldsymbol{d}$, Dynamic adhesion maps generated from time-lapse images of the growth cone in $\boldsymbol{a}$ turning toward the BDNF gradient. Consecutive $3 \mathrm{~min}$ time periods were used (18 images each) to create adhesion maps. Point contacts are labeled with thick arrows, while long arrows indicate the proximal versus distal sides of the growth cone, as well as the direction of outgrowth. Note that a greater number of point contacts with a shorter lifetimes form proximal to the gradient. $\boldsymbol{e}-\boldsymbol{g}$, Three time-point merged images pseudocolored red, green, and blue from the neuron in $\boldsymbol{a}$ - $\boldsymbol{d}$ illustrate filopodial initiation and extension. Note an increased number of PY-positive filopodial tips toward BDNF. $\boldsymbol{h}, \boldsymbol{i}$, Quantification of asymmetric point contact dynamics across growth cones during turning. $\boldsymbol{j}, \boldsymbol{k}$, Quantification of asymmetric PY filopodial tip dynamics. Significance determined by Student's $t$ test. $n=5$ neurons for $\boldsymbol{h}-\boldsymbol{i}$ and $\boldsymbol{j}-\boldsymbol{k}$. Note that point contact dynamics were quantified from paxillin-GFPexpressing neurons. ${ }^{* * *} p<0.001,{ }^{* *} p<0.01$. Scale bar, $5 \mu \mathrm{m}$

side was also over twice as high as the distal side (Fig. 8i), which we found to be $1.28 \pm 0.27$ point contacts per minute proximally versus $0.57 \pm 0.17$ distally. To eliminate any potential bias based on preselection of turning versus nonturning growth cones, we also analyzed the entire set of neurons tested. Proximal-to-distal point contact lifetimes across all growth cones examined was $134.6 \pm 4.7$ versus $157.5 \pm 5.2 \mathrm{~s}(p<0.05)$, whereas proximalto-distal point contact frequencies for all growth cones was $0.99 \pm 0.16$ versus $0.68 \pm 0.10(p<0.05)$. As an additional measure of the local response to BDNF, we quantified phosphotyrosine signals at filopodial tips of responsive neurons by $\mathrm{mCH}-$ dSH2 fluorescence. The filopodial tip response was much stronger on the proximal side, illustrated by three time-point merged images (Fig. $8 e-g, j)$. Twice as many PY-containing tips were observed on the proximal side of growth cones (Fig. $8 k$ ), revealing enhanced filopodial formation, as well as higher PY signals at tips facing the gradient. Together, these results show that BDNF attraction involves the differential regulation of filopodia, lamellipodia, and point contacts across growth cones.

\section{Discussion}

Here we identify a link between the modulation of integrindependent adhesive contacts and chemotropic axon guidance. We show that an attractive guidance cue, BDNF, stimulates growth cone point contact formation and turnover. We find that BDNF drives axon outgrowth and guidance through phosphor-

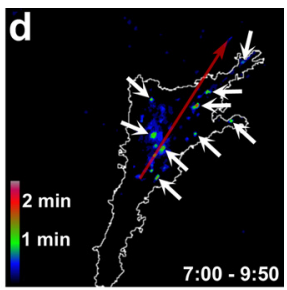

ylation of FAK at key residues that influence distinct and indispensable aspects of growth cone motility. FAK phosphorylation at Y861 is essential for lamellipodial protrusion induced by BDNF, while phosphorylation at Y925 controls the rate of point contact turnover. Each of these signals is necessary for attractive turning to BDNF in vitro, emphasizing the importance of protrusion and adhesion for axon guidance. Importantly, we showed that point contacts are differentially regulated within growth cones during turning, suggesting that control of growth cone adhesion may be necessary for axon pathfinding in vivo.

In motile cells, FAK is known to integrate signals generated from both soluble and substratum-associated cues, which feed forward through several downstream targets to control various aspects of cell motility (Mitra et al., 2005). FAK is activated by autophosphorylation at Y397 upon integrin receptor clustering or by growth factor receptors. Subsequently, Src family kinases are activated by binding to pY-397 FAK, which leads to Srcmediated phosphorylation of FAK at additional tyrosine residues. Previously, we showed that active Src family kinases promote filopodial extension through tyrosine phosphorylation of protein(s) at filopodial tips (Robles et al., 2005). Interestingly, we find here that FAK function is also required for stimulation of PY at filopodial tips by BDNF (Fig. 3), yet FAK itself does not target to the tips of extending filopodia (Fig. $3 h$ ). This result suggests that FAK may activate Src at adhesion sites, then release it to target proteins at the tips of filopodia. Other phosphorylated tyrosine residues on FAK recruit different downstream effectors. Phosphorylation of FAK Y861 allows binding of p130Cas and signaling to Rac1, which promotes lamellipodial protrusion (Cho and Klemke, 2002). p130Cas has previously been shown to function downstream of Netrin-mediated axon guidance, and upstream of Rac1 and Cdc42 (Liu et al., 2007). Here we find that expression of nonphosphorylatable Y861F specifically prevents lamellipodial protrusion induced by BDNF (Fig. 6), as well as attractive guidance in vitro (Fig. 7). Therefore, similar to Src, p130Cas may be activated by FAK in response to BDNF at adhesion sites, but function distally at the leading edge to promote veil protrusion.

While FAK is one of the first proteins recruited to adhesions, it is not necessary for adhesion formation, since $\mathrm{FAK}^{-1-}$ fibroblasts form robust adhesions (Ilić et al., 1995). However, adhesions in $\mathrm{FAK}^{-1-}$ fibroblasts are longer lived and do not undergo the normal maturation process, implicating FAK in focal adhesion turnover, but not formation. Point contacts in FAK loss of function growth cones are also longer lived in both RGCs (Woo et al., 2009) and spinal neurons (Fig. 4). FAK may have a role in adhesion assembly as well, as work in mouse embryonic fibroblasts showed that the FAK homolog Pyk2 is upregulated when FAK function is lost (Lim et al., 2008). Pyk2 knockdown in FAK- 
null fibroblasts resulted in a marked loss of adhesions, suggesting that Pyk2 may be sufficient for adhesion formation, but not turnover. It is possible that Pyk2 is playing a similar role in neurons, partially compensating for loss of FAK function. We find that Pyk2 is present within whole Xenopus embryos at the neural tube stage, but cannot detect Pyk2 mRNA by RT-PCR from pure spinal cord (data not shown). However, it is possible that Pyk2 is upregulated in FAK LOF spinal neurons.

Several FAK-related signaling pathways can affect adhesion turnover, indicating that a complex interplay of signals controls adhesion dynamics (Mitra et al., 2005). For example, FAK Y925Fexpressing cells exhibit delayed adhesion turnover (Brunton et al., 2005). We also find that Y925F FAK inhibits growth cone point contact turnover (Fig. 6), as well as growth cone turning toward BDNF (Fig. 7). Phosphorylation of Y925 FAK creates a binding site for several effectors, including the growth factor regulatory element Grb2. Because Y925 overlaps with the paxillinbinding domain of FAK, it has been speculated that Grb2 association with FAK will displace FAK from paxillin and allow adhesion turnover (Mitra et al., 2005; Scheswohl et al., 2008). The distinct phenotypes we observe between Y925F and the Y861F mutants are noteworthy, as both pathways are necessary for attractive turning on LN in vitro (Fig. 7), it appears that FAK coordinates growth cone protrusion and adhesion downstream of BDNF and integrin engagement.

One key set of effectors that FAK regulates are the Rho family GTPases, which control motility and adhesion of migrating cells and growth cones (Dickson, 2001; Etienne-Manneville and Hall, 2002). In previous work, we demonstrated that Rac-1 activity is necessary for the formation of small, labile point contacts and that RhoA activity promotes point contact stabilization (Woo and Gomez, 2006). Here we chose to limit our analysis to the longer-lived, presumably RhoA-dependent population. Increased point contact turnover with BDNF may be due to a decrease in RhoA activity. One principal target of FAK phosphorylation is paxillin, which leads to inhibition of RhoA through p190Rho GAP (Turner, 2000). Additionally, BDNF is known to reduce RhoA activity in growth cones in a p75 ${ }^{\text {NTR }}$-dependent manner (Gehler et al., 2004). The overstabilization we observe in FAK LOF growth cones may be due to unregulated RhoA activity, a correlation supported by studies of FAK-null fibroblasts (Tomar et al., 2009). The lack of an adhesion phenotype seen in the Y861F mutant, which we hypothesize links to p130Cas-Rac1, implies that basal levels of Rac activity are present independent of FAK function.

FAK has been shown to function downstream of a number of both positive and negative axon guidance cues, including BDNF, Netrin, Sema3A, repulsive guidance molecule, myelin-associated glycoprotein (MAG), chondroitin sulfate proteoglycan (CSPG), and Eph/Ephrins (Li et al., 2004; Liu et al., 2004; Ren et al., 2004; Woo and Gomez, 2006; Parri et al., 2007; Bechara et al., 2008; Goh et al., 2008; Endo and Yamashita, 2009; Shi et al., 2009; Tan et al., 2011). How FAK functions as a common target of such diverse axon guidance cues is not known. In the case of Netrin and our results with BDNF, FAK activation and its extended phosphorylation regulate multiple signaling pathways that promote motility (Li et al., 2004; Liu et al., 2004; Ren et al., 2004). In contrast, CSPG inhibits axon outgrowth by inhibiting integrin activation of FAK (Tan et al., 2011). Complicating matters, some guidance cues that inhibit motility also activate FAK. For example, EphrinA1 activates FAK but induces point contact stabilization and stalled outgrowth (Woo et al., 2009). However, the inhibitory cues MAG and Sema3A lead to FAK activation and point contact disassembly (Woo and Gomez, 2006; Bechara et al.,
2008). Although these results may appear contradictory, it should be emphasized that FAK likely functions cyclically and not simply as an "on-off" switch. We hypothesize that optimal axon outgrowth on integrin-activating ECM proteins occurs when FAK can rapidly cycle between an active and inactive state. Thus, factors that disrupt cycling can inhibit outgrowth by either stabilizing or destabilizing adhesions. It is also possible that different guidance cues promote differential phosphorylation of FAK at its various tyrosine residues, which have distinct signaling outputs. Finally, it is clear that axon guidance cues activate multiple parallel signaling pathways; for example, RhoA activation by Ephrins through ephexin may modulate the effects of FAK activation by integrins.

Several pieces of evidence suggest that growth cone point contacts can be instructive in axon guidance. Previously we showed that FAK activity is necessary for midline crossing by commissural interneurons, as well as proper arborization by RohanBeard sensory neurons in the skin (Robles and Gomez, 2006). Additionally, a loss of FAK function resulted in topographic mapping errors in RGCs projecting within the tectum (Woo et al., 2009). A recent publication showed that integrin receptors are differentially endocytosed on exposure to a gradient of the repulsive cue MAG (Hines et al., 2010). Here we present direct evidence that adhesive point contacts are differentially distributed and have varying turnover rates during turning toward BDNF (Fig. 8). These results, along with our data that FAK-mediated control of adhesion is necessary for turning (Fig. 7), provide strong evidence that regulation of point contacts is a key process in axon guidance. A growing axon in the developing embryo integrates a diverse array of cues using an interlinked network of intracellular signals. Growth cone behavior is modulated by these signals through regulation of lamellipodial and filopodial dynamics and, from our data, adhesion to its environment. Differential adhesion dynamics allow for attraction to a cue across the $\mathrm{ECM}$, and repulsion involves reciprocal asymmetry in point contact disassembly (Hines et al., 2010). Indeed, a loss of adhesion is part of the collapse response seen with Sema3a and high doses of EphrinA1 (Bechara et al., 2008; Woo et al., 2009). Our results show that adhesion dynamics need to be considered along with protrusion and other processes to fully understand the mechanisms allowing for axon guidance.

\section{References}

Bechara A, Nawabi H, Moret F, Yaron A, Weaver E, Bozon M, Abouzid K Guan JL, Tessier-Lavigne M, Lemmon V, Castellani V (2008) FAKMAPK-dependent adhesion disassembly downstream of L1 contributes to semaphorin3A-induced collapse. EMBO J 27:1549-1562.

Bouton AH, Riggins RB, Bruce-Staskal PJ (2001) Functions of the adapter protein Cas: signal convergence and the determination of cellular responses. Oncogene 20:6448-6458.

Brunton VG, Avizienyte E, Fincham VJ, Serrels B, Metcalf CA 3rd, Sawyer TK, Frame MC (2005) Identification of Src-specific phosphorylation site on focal adhesion kinase: dissection of the role of Src SH2 and catalytic functions and their consequences for tumor cell behavior. Cancer Res 65:1335-1342.

Cary LA, Chang JF, Guan JL (1996) Stimulation of cell migration by overexpression of focal adhesion kinase and its association with Src and Fyn. J Cell Sci 109:1787-1794.

Cho SY, Klemke RL (2002) Purification of pseudopodia from polarized cells reveals redistribution and activation of Rac through assembly of a CAS/ Crk scaffold. J Cell Biol 156:725-736.

Dent EW, Meiri KF (1992) GAP-43 phosphorylation is dynamically regulated in individual growth cones. J Neurobiol 23:1037-1053.

Dickson BJ (2001) Rho GTPases in growth cone guidance. Curr Opin Neurobiol 11:103-110.

Endo M, Yamashita T (2009) Inactivation of Ras by p120GAP via focal ad- 
hesion kinase dephosphorylation mediates RGMa-induced growth cone collapse. J Neurosci 29:6649-6662.

Etienne-Manneville S, Hall A (2002) Rho GTPases in cell biology. Nature 420:629-635.

Gehler S, Gallo G, Veien E, Letourneau PC (2004) p75 neurotrophin receptor signaling regulates growth cone filopodial dynamics through modulating RhoA activity. J Neurosci 24:4363-4372.

Goh EL, Young JK, Kuwako K, Tessier-Lavigne M, He Z, Griffin JW, Ming GL (2008) Betal-integrin mediates myelin-associated glycoprotein signaling in neuronal growth cones. Mol Brain 1:10.

Gomez TM, Roche FK, Letourneau PC (1996) Chick sensory neuronal growth cones distinguish fibronectin from laminin by making substratum contacts that resemble focal contacts. J Neurobiol 29:18-34.

Gomez TM, Harrigan D, Henley J, Robles E (2003) Working with Xenopus spinal neurons in live cell culture. Methods Cell Biol 71:129-156.

Hamadi A, Bouali M, Dontenwill M, Stoeckel H, Takeda K, Rondé P (2005) Regulation of focal adhesion dynamics and disassembly by phosphorylation of FAK at tyrosine 397. J Cell Sci 118:4415-4425.

Hines JH, Abu-Rub M, Henley JR (2010) Asymmetric endocytosis and remodeling of betal-integrin adhesions during growth cone chemorepulsion by MAG. Nat Neurosci 13:829-837.

Huber AB, Kolodkin AL, Ginty DD, Cloutier JF (2003) Signaling at the growth cone: ligand-receptor complexes and the control of axon growth and guidance. Annu Rev Neurosci 26:509-563.

Hynes RO (2002) Integrins: bidirectional, allosteric signaling machines. Cell 110:673-687.

Ilić D, Furuta Y, Kanazawa S, Takeda N, Sobue K, Nakatsuji N, Nomura S, Fujimoto J, Okada M, Yamamoto T (1995) Reduced cell motility and enhanced focal adhesion contact formation in cells from FAK-deficient mice. Nature 377:539-544.

Kirchner J, Kam Z, Tzur G, Bershadsky AD, Geiger B (2003) Live-cell monitoring of tyrosine phosphorylation in focal adhesions following microtubule disruption. J Cell Sci 116:975-986.

Letourneau PC, Condic ML, Snow DM (1994) Interactions of developing neurons with the extracellular matrix. J Neurosci 14:915-928.

Li W, Lee J, Vikis HG, Lee SH, Liu G, Aurandt J, Shen TL, Fearon ER, Guan JL, Han M, Rao Y, Hong K, Guan KL (2004) Activation of FAK and Src are receptor-proximal events required for netrin signaling. Nat Neurosci 7:1213-1221.

Lim Y, Han I, Jeon J, Park H, Bahk YY, Oh ES (2004) Phosphorylation of focal adhesion kinase at tyrosine 861 is crucial for Ras transformation of fibroblasts. J Biol Chem 279:29060-29065.

Lim Y, Lim ST, Tomar A, Gardel M, Bernard-Trifilo JA, Chen XL, Uryu SA, Canete-Soler R, Zhai J, Lin H, Schlaepfer WW, Nalbant P, Bokoch G, Ilic D, Waterman-Storer C, Schlaepfer DD (2008) PyK2 and FAK connections to p190Rho guanine nucleotide exchange factor regulate RhoA activity, focal adhesion formation, and cell motility. J Cell Biol 180:187-203.

Liu G, Beggs H, Jürgensen C, Park HT, Tang H, Gorski J, Jones KR, Reichardt LF, Wu J, Rao Y (2004) Netrin requires focal adhesion kinase and Src family kinases for axon outgrowth and attraction. Nat Neurosci 7:1222-1232.

Liu G, Li W, Gao X, Li X, Jürgensen C, Park HT, Shin NY, Yu J, He ML, Hanks SK, Wu JY, Guan KL, Rao Y (2007) p130CAS is required for netrin signaling and commissural axon guidance. J Neurosci 27:957-968.

Lowery LA, Van Vactor D (2009) The trip of the tip: understanding the growth cone machinery. Nat Rev Mol Cell Biol 10:332-343.

Ming G, Lohof AM, Zheng JQ (1997a) Acute morphogenic and chemotropic effects of neurotrophins on cultured embryonic Xenopus spinal neurons. J Neurosci 17:7860-7871.

Ming GL, Song HJ, Berninger B, Holt CE, Tessier-Lavigne M, Poo MM (1997b) cAMP-dependent growth cone guidance by netrin-1. Neuron 19:1225-1235.

Mitra SK, Hanson DA, Schlaepfer DD (2005) Focal adhesion kinase: in command and control of cell motility. Nat Rev Mol Cell Biol 6:56-68.
Nieuwkoop PD, Faber J (1994) Normal table of Xenopus laevis (Daudin). New York: Garland.

Okada M, Nakagawa H (1989) A protein tyrosine kinase involved in regulation of pp60c-src function. J Biol Chem 264:20886-20893.

Parri M, Buricchi F, Giannoni E, Grimaldi G, Mello T, Raugei G, Ramponi G, Chiarugi P (2007) EphrinAl activates a Src/focal adhesion kinasemediated motility response leading to rho-dependent actino/myosin contractility. J Biol Chem 282:19619-19628.

Renaudin A, Lehmann M, Girault J, McKerracher L (1999) Organization of point contacts in neuronal growth cones. J Neurosci Res 55:458-471.

Ren XR, Ming GL, Xie Y, Hong Y, Sun DM, Zhao ZQ, Feng Z, Wang Q, Shim S, Chen ZF, Song HJ, Mei L, Xiong WC (2004) Focal adhesion kinase in netrin-1 signaling. Nat Neurosci 7:1204-1212.

Robles E, Gomez TM (2006) Focal adhesion kinase signaling at sites of integrin-mediated adhesion controls axon pathfinding. Nat Neurosci 9:1274-1283.

Robles E, Woo S, Gomez TM (2005) Src-dependent tyrosine phosphorylation at the tips of growth cone filopodia promotes extension. J Neurosci 25:7669-7681.

Santiago-Medina M, Myers JP, Gomez TM (2011) Imaging adhesion and signaling dynamics in Xenopus laevis growth cones. Dev Neurobiol. Advance online publication. doi:10.1002/dneu.20886.

Schaller MD (2010) Cellular functions of FAK kinases: insight into molecular mechanisms and novel functions. J Cell Sci 123:1007-1013.

Schaller MD, Borgman CA, Parsons JT (1993) Autonomous expression of a noncatalytic domain of the focal adhesion-associated protein tyrosine kinase pp125FAK. Mol Cell Biol 13:785-791.

Schaller MD, Hildebrand JD, Shannon JD, Fox JW, Vines RR, Parsons JT (1994) Autophosphorylation of the focal adhesion kinase, pp125FAK, directs SH2-dependent binding of pp60src. Mol Cell Biol 14:1680-1688.

Scheswohl DM, Harrell JR, Rajfur Z, Gao G, Campbell SL, Schaller MD (2008) Multiple paxillin binding sites regulate FAK function. J Mol Signal 3:1.

Shi Y, Pontrello CG, DeFea KA, Reichardt LF, Ethell IM (2009) Focal adhesion kinase acts downstream of EphB receptors to maintain mature dendritic spines by regulating cofilin activity. J Neurosci 29:8129-8142.

Tan CL, Kwok JC, Patani R, Ffrench-Constant C, Chandran S, Fawcett JW (2011) Integrin activation promotes axon growth on inhibitory chondroitin sulfate proteoglycans by enhancing integrin signaling. J Neurosci 31:6289-6295.

Tessier-Lavigne M, Goodman CS (1996) The molecular biology of axon guidance. Science 274:1123-1133.

Thomas SM, Brugge JS (1997) Cellular functions regulated by Src family kinases. Annu Rev Cell Dev Biol 13:513-609.

Tomar A, Schlaepfer DD (2009) Focal adhesion kinase: switching between GAPs and GEFs in the regulation of cell motility. Curr Opin Cell Biol 21:676-683.

Tomar A, Lim ST, Lim Y, Schlaepfer DD (2009) A FAK-p120RasGAPp190RhoGAP complex regulates polarity in migrating cells. J Cell Sci 122:1852-1862.

Turner CE (2000) Paxillin and focal adhesion signalling. Nat Cell Biol 2:E231-E236.

Woo S, Gomez TM (2006) Racl and RhoA promote neurite outgrowth through formation and stabilization of growth cone point contacts. J Neurosci 26:1418-1428.

Woo S, Rowan DJ, Gomez TM (2009) Retinotopic mapping requires focal adhesion kinase-mediated regulation of growth cone adhesion. J Neurosci 29:13981-13991.

Yaron A, Zheng B (2007) Navigating their way to the clinic: emerging roles for axon guidance molecules in neurological disorders and injury. Dev Neurobiol 67:1216-1231.

Yu TW, Bargmann CI (2001) Dynamic regulation of axon guidance. Nat Neurosci 4 [Suppl]:1169-1176. 